\title{
Master-Equation Approach to the Glass Transition
}

\author{
Jeppe C. Dyre \\ Institute of Mathematics and Physics IMFUFA, University of Roskilde, DK-4000 Roskilde, Denmark
}

(Received 13 November 1986)

\begin{abstract}
From a simple master-equation description of viscous liquids it is shown that there exist two different kinds of glass transitions. Slow glass transitions lead to a Gaussian, while fast glass transitions lead to an exponential, distribution of frozen-in energies in the glass. It is argued that amorphous semiconductors prepared by a fast glass transition have exponential band tails of localized states.
\end{abstract}

PACS numbers: $64.70 . P f$

By sufficiently rapid cooling, glasses may be formed from any liquid, whether bonded by covalent, ionic, metallic, or molecular forces. The glass transition is thus a universal phenomenon. ${ }^{1-6}$ It continues to attract attention both from a purely scientific and from a practical point of view. As a fundamental physical problem, the glass transition is interesting because it somehow involves a breakdown of ergodicity. ${ }^{7}$ Technologically, an understanding of the glass transition is important because glass properties depend on exactly how the liquid structure is frozen at the glass transition.

Since glasses are formed from viscous liquids, a better understanding of glasses and the glass transition must derive from a better understanding of viscous liquids. The properties of simple liquids can be calculated from first principles today, ${ }^{8,9}$ but this is not the case for viscous liquids. Though recently there have been interesting attempts to extrapolate the theory of simple liquids into the viscous and glassy regime, ${ }^{10,11}$ we here take the different point of view in which viscous liquids are regarded as qualitaticely different from nonviscous liquids. The idea is the following. A high viscosity implies a small diffusion constant. This means that most molecular motion goes into vibrations so that a viscous liquid spends most time in potential-energy minima. ${ }^{12}$ Occasionally, effective displacements of the molecules do take place, however. Since one molecule cannot move without having its neighbors move too, these "flow events" must be highly cooperative. This is an old idea; the small parts of the viscous liquid in which the flow events take place have been referred to as "cooperatively rearranging regions," 13 "quasi-independent units," 14 "thermokinetic structures," 15 or just "regions."16 This picture of viscous flow is simple and attractive. There is evidence in favor of it from extensive computer simulations. ${ }^{5}$ Also, from a comparison of dielectric relaxation and Kerr-effect measurements it can be shown that dipolar reorientation in viscous liquids occurs cooperatively via large-angle jumps and does not take place by rotational diffusion. ${ }^{17,18}$

The cooperatively rearranging regions are believed to be fairly small but large enough to be considered as mutually noninteracting. The viscous liquid may therefore be regarded as an ensemble of regions, each of which spends most time in a potential-energy minimum. Henceforth, potential energy is referred to as energy and energy minima as states. Now, to describe the dynamics of the regions, it is natural to use transition-state theory. ${ }^{14,19}$ Goldstein has suggested that the transition state corresponds to the high-temperature, more-fluid, liquid. ${ }^{14}$ If we denote the energy of this "fluid" state by $E_{0}$, the relaxation time, $\tau(E)$, for transitions from a state with energy $E$ is given by

$$
\tau(E)=\tau_{0} e^{\left(E_{0}-E\right) / T} \quad\left(E<E_{0}\right),
$$

where $\tau_{0}$ is a microscopic time and Boltzmann's constant is put equal to unity. In a recent very interesting paper Brawer has shown that Eq. (1) in the region picture is able to explain all observed features of relaxation in viscous liquids and glasses. ${ }^{16}$ These features include the nonlinearity, the broad distributions of relaxation times, and the fact that glassy relaxation proceeds with a smaller activation energy than relaxation in viscous liquids.

The "fluid" state must have a structure much different from the lower-lying states. It is reasonable to assume that, once excited into this state, a region has forgotten which state it came from and may end up in any other state. This is consistent with the observed large-angle dipolar reorientations. ${ }^{18}$ Under this assumption, a simple master equation describes the time evolution of the energy probability density, $P(E, t)$ :

$$
\frac{\partial P(E, t)}{\partial t}=-\frac{P(E, t)}{\tau(E)}+n(E) \int_{0}^{E_{0}} \frac{P\left(E^{\prime}, t\right)}{\tau\left(E^{\prime}\right)} d E^{\prime}
$$

where $\tau$ is given by Eq. (1), $n(E)$ is the normalized density of states, and the lowest possible region energy is zero. Equation (2) is equivalent to Brawer's kinetic equation. ${ }^{16}$

Since each region consists of many molecules, thermodynamic concepts may be applied. ${ }^{3}$ In thermal equilibrium, $P(E, t)$ is approximately a Gaussian, centered around the mean energy $\bar{E}(T)$. If the temperature is lowered, the Gaussian is displaced towards lower energies. For a finite cooling rate, however, the system will fall out of equilibrium sooner or later for continued cooling. This is, of course, the glass transition; it takes place around the temperature where the time to relax to equi- 
librium is comparable to the cooling time. Suppose the system is cooled at a constant rate to zero temperature in time $t_{0}$, from a state of thermal equilibrium at high temperature where the liquid relaxation time is small compared to $t_{0}$. At any temperature during the cooling the equation $\tau(E)=t_{0}$ defines a characteristic energy, $E_{d}$, given by

$$
E_{d}=E_{0}-T \ln \left(t_{0} / \tau_{0}\right)
$$

Regions with energy less than $E_{d}$ are frozen. $E_{d}$, the so-called demarcation energy, was originally introduced in the theory for excited charge-carrier thermalization in amorphous semiconductors. ${ }^{20,21}$ As the temperature is lowered, $E_{d}$ increases while at the same time the equilibrium Gaussian is displaced towards lower energies. When the Gaussian meets $E_{d}$ the glass transition takes place.

We have studied the glass transition for systems with constant specific heat $c$. The solution of Eq. (2) is plotted in Fig. 1 for $c=3,9,27,81$ at a cooling rate given by $\ln \left(t_{0} / \tau_{0}\right)=9$. The full curve is $P(E, t)$, the dotted curve is the equilibrium $P(E)$, and the vertical line marks $E_{d}$. For each value of $c$ four snapshots are given, starting at almost thermal equilibrium and ending showing the frozen-in energy distribution which will be denoted by $P_{0}(E)$. Figure 1 gives an idea of the physics of the glass transition according to Eq. (2). The figure shows that $P_{0}(E)$ is essentially a Gaussian for small c's while it is asymmetric for large $c$ values.

It is not hard to understand what happens at the glass transition in two limiting cases. Consider first small cooling rates: $\ln \left(t_{0} / \tau_{0}\right) \gg c$. In this case $E_{d}$ moves fast towards higher energies while the equilibrium Gaussian almost does not move at all. When $E_{d}$ sweeps past the Gaussian it simply freezes it, so that $P_{0}(E)$ is just the equilibrium Gaussian at the glass transition temperature, $T_{g}$ :

$$
P_{0}(E) \cong\left[2 \pi\left\langle(\Delta E)^{2}\right\rangle\right]^{-1 / 2} \exp \left[-\frac{\left(E-E_{g}\right)^{2}}{2\left\langle(\Delta E)^{2}\right\rangle}\right] \quad \text { (slow cooling), }
$$

where $E_{g}=c T_{g},\left\langle(\Delta E)^{2}\right\rangle=c T_{g}^{2}$, and $T_{g}$ is determined from $E_{d}\left(T_{g}\right)=c T_{g}$, i.e.,

$$
T_{g}=E_{0} /\left[c+\ln \left(t_{0} / \tau_{0}\right)\right] \text {. }
$$

In the case of fast cooling rates, $\ln \left(t_{0} / \tau_{0}\right) \ll c$, something different happens. Then $E_{d}$ moves only very slowly compared to the Gaussian, so that $E_{d}$ is almost constant during the glass transition. Approaching the glass transition, the regions jump in energy according to Eq. (2) until they, with probability $\propto n(E)$, happen to hit an energy $E$ below $E_{d}$. There will be only a few states left above $E_{g}=c T_{g}$ in the glassy state, where the glass transition temperature is again given by Eq. (5). Since $n(E)$ is approximately exponential around $E_{g}$ with a slope of $1 / T_{g}$, we find

$$
P_{0}(E) \cong\left\{\begin{array}{l}
T_{g}^{-1} \exp \left[\left(E-E_{g}\right) / T_{g}\right], \quad E<E_{g} \\
0, \quad E>E_{g} \quad \text { (fast cooling) }
\end{array}\right.
$$

An illustration of this result is provided by the inset of the $c=81$ case in Fig. 1. There will, of course, always be some states left above $E_{g}$; note also that $E_{g} \rightarrow E_{0}$ as $c \rightarrow \infty$.

We conclude that, insofar as Eq. (2) does describe viscous liquids, there exist two different kinds of glass transitions: slow and fast glass transitions. Since glasses are thermally arrested liquids, it is generally believed that the glass structure is nearly the same as the structure of the equilibrium liquid at temperatures just above $T_{g}$. According to the present theory, however, this is true only if the glass is prepared by a slow glass transition. Curiously enough, a slow glass transition freezes the equilibrium structure because $E_{d}$ moves fast across the equilibrium Gaussian.
The relevant parameter characterizing the glass transition is the ratio $K=\ln \left(t_{0} / \tau_{0}\right) / c_{g}$. Here $c_{g}$ is the region specific heat at $T_{g}$, allowing for the more general case of a temperature-dependent specific heat. The region energies are distributed according to a Gaussian or an exponential, depending on whether $K \gg 1$ or $K \ll 1$. This has important consequences for the properties of glasses. By expanding to first order, one finds that any physical property which is a function of $E$ will be Gaussian or exponentially distributed depending on the value of $K$. This may explain the ubiquitous appearance of Gaussian and exponential energy-barrier distributions for linearly and nonlinearly relaxing degrees of freedom in glassy solids. ${ }^{22-25}$ For instance, amorphous semiconductors prepared by a fast glass transition are likely to have exponential band tails: It is reasonable to assume that the region energy is a function of density, where low densities correspond to large energies. ${ }^{16}$ The transfer integral, $t$, for electron jumps depends exponentially on the distance between neighboring atoms, but for sufficiently small distance fluctuations $t$ may be expanded to first order. Expanding also the region energy $E$ to first order in $\bar{r}$, the average atom-atom distance within a region, one finds from Eq. (6) for the distribution of average transfer integrals within a region $p(\bar{t}) \propto \exp \left(-\bar{t} / T_{0}\right)$, where $T_{0}=T_{g}(d \bar{r} / d E)|d \bar{t} / d \bar{r}|$. This implies an exponentially decreasing distribution of band widths for the bands of electron states within each region. With allowance for electron jumps between the regions, the midband states will delocalize but the tail states will probably remain localized within each region. It is not hard to show then that the bulk solid will have exponential band tails of localized states. 

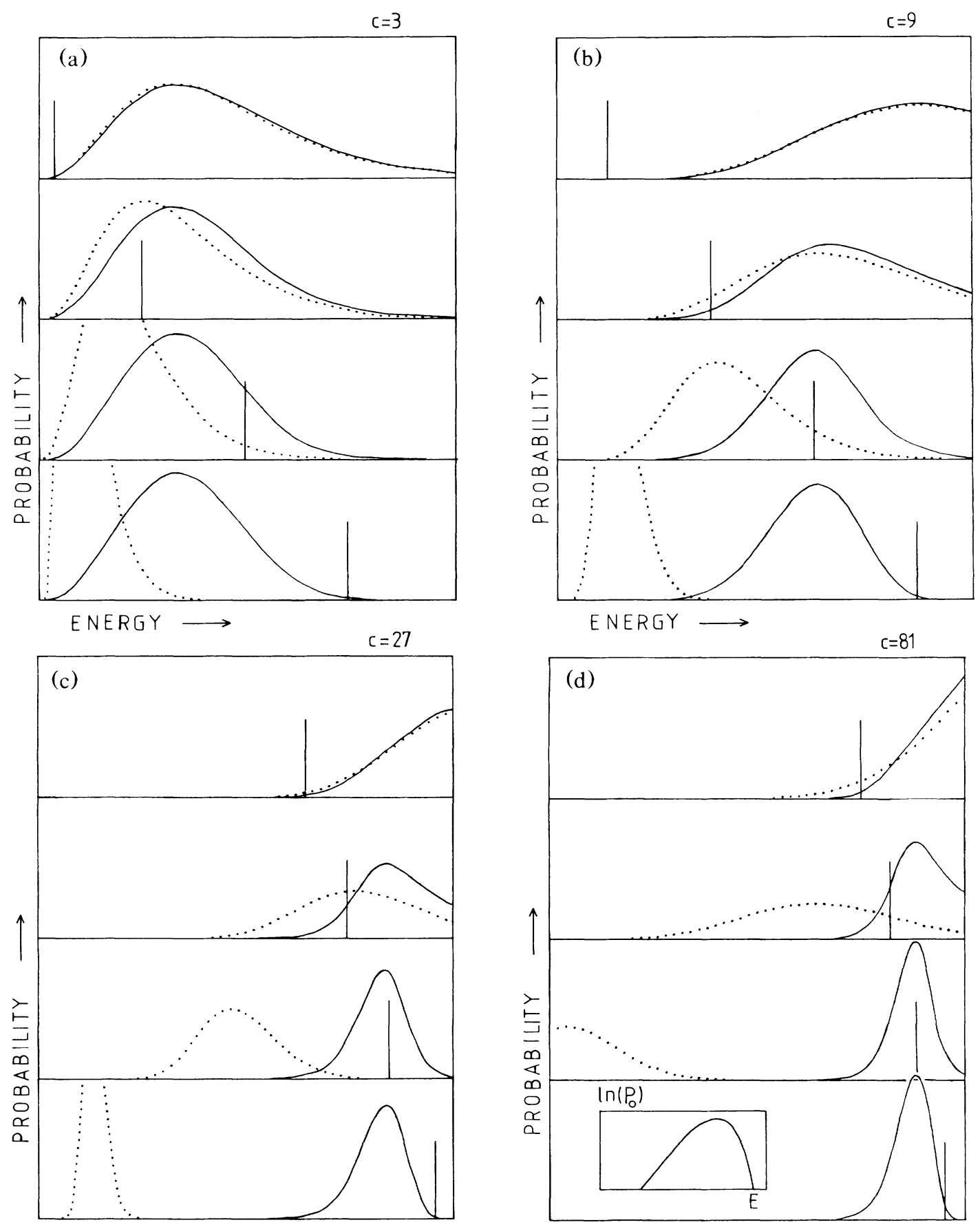

ENERGY $\longrightarrow$

ENERGY $\longrightarrow$

FIG. 1. Solution of Eq. (2) showing the glass transition for various values of the specific heat of the cooperatively rearranging regions in the viscous liquid. The cooling time, $t_{0}$, is given by $\ln \left(t_{0} / \tau_{0}\right)=9$. The full curve is $P(E, t)$, the dotted curve is the thermal equilibrium energy probability density which is approximately Gaussian, and the line shows the demarcation energy $E_{d}$. All states below $E_{d}$ are frozen. The glass transition takes place when, upon cooling, $E_{d}$ meets the equilibrium Gaussian. For each value of $c$, four snapshots of the cooling are shown, the lowest subfigure showing the frozen-in energy distribution, $P_{0}(E)$. Figure 1 is meant to illustrate Eqs. (4) and (6) according to which $P_{0}(E)$ is a Gaussian for slow cooling $\left[c \ll \ln \left(t_{0} / \tau_{0}\right)\right]$ and an exponential for fast cooling $\left[c \gg \ln \left(t_{0} / \tau_{0}\right)\right]$. If $T_{s}$ denotes the starting temperature for the cooling process, the parameters for the figure are, for $c=3$, $T_{s}=0.111 E_{0}, 0<E<E_{0} ;$ for $c=9, T_{s}=0.097 E_{0}, 0<E<E_{0} ;$ for $c=27, T_{s}=0.045 E_{0}, 0<E<E_{0} ;$ for $c=81, T_{s}=0.020 E_{0}$, $E_{0} / 2<E<E_{0}$. 
The present approach to the glass transition differs in important respects from that of Adam and Gibbs. ${ }^{13}$ In their model, the size of the regions is temperature dependent and this is ultimately the cause of the slow relaxation near the glass transition. Also, it is assumed that each region only contains a few states, while here a region has many available states. Despite these differences, the present theory is not necessarily inconsistent with the beautiful idea of an underlying secondorder phase transition to a state of zero configurational entropy at a finite temperature. ${ }^{26}$ As shown by Goldstein, even a finite region may have a rather sharp transition to a state of almost zero entropy. ${ }^{14}$ There will be a transition at $T=T_{0}$ if $n(E) \propto \exp \left(E / T_{0}\right)$ as $E \rightarrow 0 . .^{14,27}$ The state of zero configurational entropy of the bulk equilibrium viscous liquid simply corresponds to having each region in its "ground state."

Throughout this Letter, by a glass transition is meant cooling from a temperature so high that the liquid relaxation time, $\tau_{s}$, is small compared to the cooling time. But another scenario is also possible, namely the cooling in a short time compared to $\tau_{s}$, e.g., by sudden cooling of a well annealed very viscous liquid. This is a true quench; obviously it leads to a Gaussian $P_{0}(E)$. The glassy state may thus be reached in three different ways: by slow glass transitions, by fast glass transitions, and by quenches. Slow glass transitions and quenches lead to a Gaussian $P_{0}(E)$, while fast glass transitions lead to an exponentially increasing, abruptly decaying $P_{0}(E)$.

The author gratefully acknowledges numerous fruitful discussions with T. Christensen and N. B. Olsen.

${ }^{1}$ W. Kauzmann, Chem. Rev. 43, 219 (1948).

${ }^{2}$ R. O. Davies and G. O. Jones, Adv. Phys. 2, 370 (1953).
${ }^{3}$ E. Donth, Glasübergang (Akademie-Verlag, Berlin, 1981).

${ }^{4}$ R. Parthasarathy, K. J. Rao, and C. N. R. Rao, Chem. Soc. Rev. 12, 361 (1983).

${ }^{5}$ S. Brawer, Relaxation in Viscous Liquids and Glasses (American Ceramic Society, Columbus, Ohio, 1985).

${ }^{6}$ J. Jäckle, Rep. Prog. Phys., to be published.

${ }^{7}$ R. G. Palmer, Adv. Phys. 31, 669 (1982).

${ }^{8}$ J. P. Hansen and I. R. McDonald, Theory of Simple Liquids (Academic, London, 1976).

${ }^{9} \mathrm{~J}$. P. Boon and S. Yip, Molecular Hydrodynamics (McGraw-Hill, New York, 1980).

${ }^{10}$ E. Leutheusser, Phys. Rev. A 29, 2765 (1984).

${ }^{11}$ U. Bengtzelius, W. Götze, and A. Sjölander, J. Phys. C 17, 5915 (1984).

${ }^{12}$ M. Goldstein, J. Chem. Phys. 51, 3728 (1969).

${ }^{13}$ G. Adam and J. H. Gibbs, J. Chem. Phys. 43, 139 (1965).

${ }^{14}$ M. Goldstein, Faraday Symp. Chem. Soc. 6, 7 (1972).

${ }^{15}$ E. Donth, J. Non-Cryst. Solids 53, 325 (1982).

${ }^{16}$ S. A. Brawer, J. Chem. Phys. 81, 954 (1984).

${ }^{17} \mathrm{G}$. Williams, in Dielectric and Related Molecular Processes, edited by M. Davies (The Chemical Society, London, 1975), Vol. 2, p. 151.

${ }^{18}$ M. S. Beevers, J. Crossley, D. C. Garrington, and G. Williams, Faraday Symp. Chem. Soc. 11, 38 (1977).

${ }^{19}$ W. Kauzmann, Rev. Mod. Phys. 14, 12 (1942).

${ }^{20}$ V. I. Arkhipov, M. S. Iovu, A. I. Rudenko, and S. D. Shutov, Phys. Status Solidi (a) 54, 67 (1979).

${ }^{21}$ J. Orenstein, M. A. Kastner, and V. Vaninov, Philos. Mag. B 46, 23 (1982).

${ }^{22}$ A. S. Nowick and B. S. Berry, IBM J. Res. Dev. 5, 297 (1961).

${ }^{23} \mathrm{~W}$. Chambron and A. Chamberod, Solid State Commun. 33, 157 (1980).

24J. R. Macdonald, J. Appl. Phys. 58, 1955, 1971 (1985).

25 J. C. Dyre, J. Phys. C 19, 5655 (1986).

${ }^{26}$ J. H. Gibbs and E. A. DiMarzio, J. Chem. Phys. 28, 373 (1958).

${ }^{27}$ B. Derrida, Phys. Rev. Lett. 45, 79 (1980). 